\title{
NUEVA ESPECIE DE PSEUDOSINELLA SCHAEFFER, 1897 DE LA PENÍNSULA IBÉRICA (COLLEMBOLA, ENTOMOBRYIDAE)
}

\author{
J. C. Simón Benito* y A. Signoret Moreno
}

\begin{abstract}
RESUMEN
Se describe una nueva especie de Pseudosinella de la Península Ibérica, P. soriensis sp. n., al mismo tiempo que se da una clave de todas las especies del grupo petterseni, al que pertenece la especie.

Palabras clave: Taxonomía, nueva especie, Península Ibérica, Pseudosinella, Entomobryidae, Collembola.
\end{abstract}

\section{ABSTRACT \\ New species of Pseudosinella Schaeffer, 1897 from the Iberian Peninsula (Collembola, Entomobryidae)}

A new species of Pseudosinella, P. soriensis sp. n. is described and a new key to worldwide species of the petterseni group, to which the new species belongs, is made.

Key words: Taxonomy, new species, Iberian Peninsula, Pseudosinella, Entomobryidae, Collembola.

\section{Introducción}

Pseudosinella petterseni Börner, 1901, es una especie descrita de Alemania caracterizada por la presencia de un grueso diente interno en el empodio, sin ojos y con espolon mazudo en el tibiotarso, Gisin en 1967 realiza una breve descripción sobre ejemplares suizos, básica para conocer su quetotaxia y más tarde Christiansen et al. (1983) dan unos suscintos caracteres en una clave en donde agrupan a todas las especies conocidas de Pseudosinella; en el año 2004 Wang, Chen, y Christiansen, redescriben esta especie sobre ejemplares chinos y proprocionan los caracteres que la redefinen, los cuales concuerdan con los datos fragmentarios dados por los autores anteriores. En el mencionado grupo se encuentran una veintena de especies distribuidas por todo el mundo y 5 de ellas (P. petterseni Börner, 1901, P. gamae Gisin, 1967, P. gajui Luciáñez y Simón, 1994, P. bachae Luciáñez y Simón, 1994, y la nueva especie $P$. soriensis sp. n.) se localizan en la Península Ibérica. Hay que hacer notar que las citas de $P$. petterseni deben considerarse como dudosas, las de Portugal corresponden (según Gisin 1967) a gamae, y las españolas habría que comprobarlas.

En el presente trabajo describimos una nueva especie de este grupo y damos la clave correspondiente para poder identificar las especies mundiales del mismo.

* Universidad Autónoma de Madrid, Facultad de Ciencias, Departamento de Biología, Comisión Docente de Zoología, C/ Darwin, $n^{\circ}$ 2, 28049 Cantoblanco-Madrid-España, e-mail: carlos.simon@uam.es 


\section{Pseudosinella soriensis sp. $\mathbf{n}$.}

LOCALIDAD TIPO. Soria, Sierra de Cabrejas, Abejar, UTM: 30TWM12, 28-04-2005; 1 ejemplar en la muestra S1P9; 4 ejemplares en la muestra S1H16; 1 ejemplar en la muestra S2P8 (holotipo $\sigma^{7}$ ), de hojarasca de sabinar Juniperus thurifera.

Todos los ejemplares están depositados en la Universidad Autónoma de Madrid, Unidad de Zoología, Laboratorio de Entomología.

DESCRIPCIÓN. Longitud de 0,7 mm. Sin pigmento en el cuerpo, carecen de omatidios. Antenas sin pigmento, su relación con la diagonal cefálica es de 1,68. La relación de los artejos antenales I:II:III:IV es 10:14:12:25. El IV artejo antenal carece de vesícula apical. Las sensilas del III órgano antenal son dos bastoncillos ligeramente doblados hacia la región interna de la antena.

Fórmula del labial: MMrELL, todas ciliadas, r es una microseda (fig. 2). La fórmula de las macrosedas dorsales es: R011/20/0201+2. La cabeza presenta R0, R1, R2, y carece de R3. Quetotaxia del II segmento abdominal: $\mathrm{pABq}_{1} \mathrm{q}_{2}$ (fig. 4). Con seda accesoria "s" junto al botriotrico anterior del IV segmento abdominal (fig. 3).

Tibiotarso con un espolón mazudo, las macrosedas internas son ciliadas y terminadas en punta. La uña con un diente mediano interno, situado en el $69,5 \%$ de la longitud interna de la uña, existe un par basal situado proximalmente, uno de ellos más desarrollado, el doble que el otro y situado en el $27,7 \%$ de dicha cresta interna, el más distal de menor tamaño se localiza en el 28,8\% de la misma. El empodio alcanza el $64 \%$ de la longitud de la cresta interna de la uña, con un gran diente interno situado en el 54\% de su longitud (fig. 1).

Tubo ventral con $5+5$ sedas basales y $12+12$ distales. Retináculo con $4+4$ dientes, sin sedas en la base. La región distal del manubrio presenta dos sedas internas y una externa. Mucrón bidentado y con una seda en su base.
DisCUsión. Pseudosinella soriensis sp. n., pertenece al grupo petterseni Börner, 1903, por carecer de corneolas y presentar un grueso diente interno en el empodio, las especies mas próximas son: bachai Luciáñez y Simón, 1994, gajui Luciáñez y Simón, 1994 y petterseni Börner, 1901, las diferencias se señalan en la Tabla 1.

Clave de especies de Pseudosinella con $0+0$ ojos y un grueso diente interno en el empodio

1. Con seda $\mathrm{S} y / 0 \mathrm{~T}$ en la cabeza …………….................... 7

- Sin sedas ni S ni T en la cabeza .......................................... 2

2. Sedas MMELL ciliadas ……......................................... 4

- Alguna o todas estas sedas lisas ........................................ 3

3. Tórax III con 2 sedas, $\mathrm{q}_{1}$ microseda ...............................

- Tórax III con 1 seda, $\mathrm{q}_{1}$ macroseda espana Christiansen, 1961

4. Abdomen II con 2 madrosedas ......................................... 6

- Abdomen II con una macroseda ........................................ 5

5. Tibiotarso con un espolón mazudo ....................................... ...................................... bachae Luciáñez y Simón, 1994 Tibiotaso sin espolón mazudo gajui Luciáñez y Simón, 1994

6. Tórax III con dos macrosedas petterseni Börner, 1901 [sensu Gisin, 1967]

- Tórax III sin macrosedas …….................. soriensis $\mathrm{sp} . \mathrm{n}$.

7. Abdomen II con mas de una macroseda ……….............. 9

- Abdomen II con una macroseda ....................................... 8

8. Tórax II con una macroseda ................................................

.......................... certa Christiansen y Bellinger, 1980

Tórax II sin macrosedas sera Christiansen y Bellinger, 1980

9. Abdomen II con 3 macrosedas ..................................... 17

- Abdomen II con 2 macrosedas ..................................... 10

10. Tórax II sin macrosedas .................................................. 11

- Tórax II con una macroseda ........... anderseni Gisin, 1967

11. Sin seda $\mathrm{p}$ en el II segmento abdominal ........................ 13

- Con seda $\mathrm{p}$ en el II segmento abdominal ...................... 12

Tabla 1.-Diferencias entre las especies del grupo petterseni mas próximas a Pseudosinella soriensis $\mathbf{s p . ~ n . ~}$

Table 1.- Differences between species of the petterseni group that are closest to Pseudosinella sorienesis $\mathbf{s p .} \mathbf{n}$.

\begin{tabular}{lccccc}
\hline Especies & Macrosedas dorsales & Labio fórmula & II Ab & s IV & Espolón \\
\hline P. bachai & $\mathrm{R} 011 / 32 / 0101+2$ & MMrELL & -aBqq & + & + \\
P. gajui & R011/32/0101+2 & MMrELL & -aBqq & + & - \\
P. petterseni & R---/22/0201+2 & MMrELL & pABqq & + & + \\
P. soriensis sp. $\mathrm{n}$. & R011/20/0201+2 & MMrELL & pABqq & + & + \\
\hline
\end{tabular}




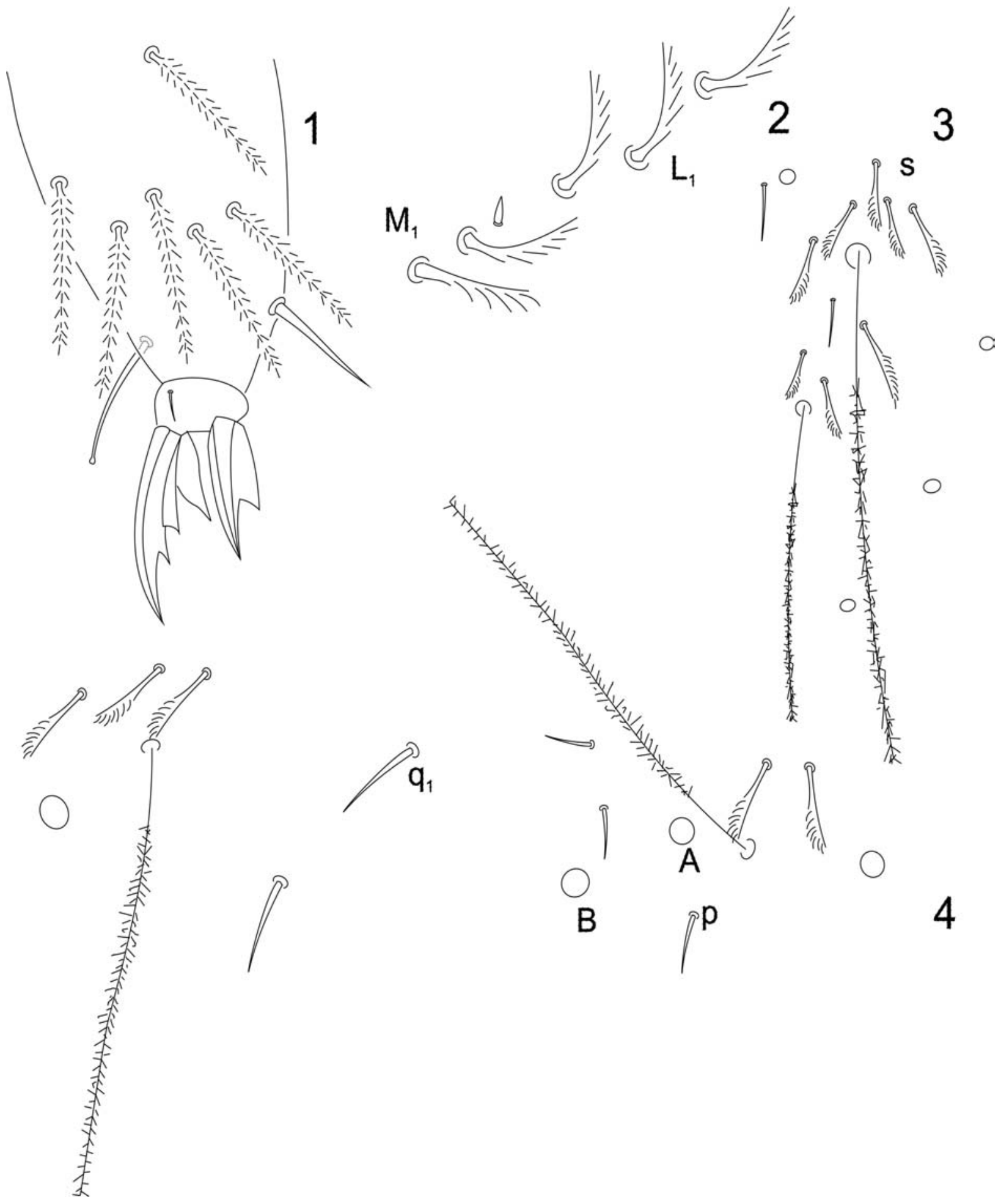

Figs. 1-4.- Pseudosinella soriensis sp. n.: 1) Uña del III par de patas. 2) Labio. 3) Quetotaxia del IV segmento abdominal. 4) Quetotaxia del II segmento abdominal.

Figs. 1-4.- Pseudosinella soriensis sp. n.: 1) Ungues of the III pair of legs. 2) Labium. 3) Chaetotaxy of Abdominal IV segment. 4) Chaetotaxy of Abdominal II segment. 
12. Seda $Q$, del II segmento abdominal macroseda rolfi Mills, 1932

- $\quad$ Seda $\mathrm{q}_{1}$ del II segmento abdominal microseda hrabrei Rusek, 1979

13. Sin sedas $\mathrm{S}$ ni $\mathrm{T}$ en la cabeza 14

- Con seda T en la cabeza biunguiculata Ellis, 1967 [sensu Mari-Mutt, 1986]

14. Sedas L del labio ciliadas

- Sedas L del labio lisas bellingeri Wang, Christiansen y Chen, 2002

15. IV segmento abdominal con $1+2$ macrosedas 16

- IV segmento abominal con 1+1 macrosedas fujiokai Yosii, 1964

16. Con seda s en el IV segmento abdominal maros Deharveng y Suhardjono, 2004

- Sin seda s en el IV segmento abdominal caoi Chen, Wang y Christiansen, 2002

17. Tibiotarso con espolón mazudo

- Tibiotarsos sin espolones mazudos. ashmoleorum Gama, 1988

18. IV segmento abdominal con $0+2$ macrosedas violenta (Folsom, 1924) [sensu Christiansen y Bellinger, 1998]

- IV segmento abdominal con 1+2 macrosedas gamae Gisin, 1967

En estas claves no se han incluido las especies Pseudosinella argentea Loksa, 1960 nec Folsom; P. halophila Bagnall, 1939 ni P. attenuata Bonnet, 1934, por carecer de datos sobre su quetotaxia.

\section{AGRADECIMIENTOS}

Queremos dar las gracias a todos los alumnos de la asignatura "Sistemas Terrestres" del curso 2004-2005 por su entusiasmo en la recogida del material, que ha dado como resultado este trabajo.

\section{Referencias}

Christiansen, K. y Bellinger, P., 1998. The Collembola of North America north of the Rio Grande. A taxonomic analysis. Updated second edition. Grinnell College. Grinnell. 1.520 pp.

Christiansen, K., Gama, M. M. \& Belllinger, P., 1983. A catalogue of the species of the genus Pseudosinella. Ciência Biológica. Ecology and Systematics, 5: 13-31.

Gisin, H., 1967. Espèces nouvelles et lignées évolutives de Pseudosinella endogés (Collembola). Memórias e Estudos do Museu Zoológico da Universidade de Coimbra, 301: 1-25.

LuCiÁnez, M. J. \& SimÓN, J. C., 1994. Cinco especies nuevas del genero Pseudosinella (Collembola: Entomobryidae) de la Península Ibérica. Annales de la Société Entomologique de France, 30: 319-327.

Mari-Mutt, J. A., 1986. Collembola associated with grasses in the grounds of the University of Puerto Rico at Mayaguez. Journal of Agriculture of the University of Puerto Rico, 70(1): 75-78.

Wang, F., Chen, J. X. \& Christiansen, K., 2004. A survey of the genus Pseudosinella (Collembola: Entomobryidaae) from East Asia. Annals of the Entomological Society of America, 97: 364-385.
Recibido, 7-VI-2006

Aceptado, 20-X-2006 Publicado, 29-XII-2006 